\title{
Re: J Child Adolesc Psychopharm 23:208-213, 2013
}

Steinhausen, Hans-Christoph ; Helenius, Dorte

DOI: https://doi.org/10.1089/cap.2014.2425

Posted at the Zurich Open Repository and Archive, University of Zurich ZORA URL: https://doi.org/10.5167/uzh-98690

Journal Article

Published Version

Originally published at:

Steinhausen, Hans-Christoph; Helenius, Dorte (2014). Re: J Child Adolesc Psychopharm 23:208-213, 2013. Journal of Child and Adolescent Psychopharmacology, 24(2):107-108.

DOI: https://doi.org/10.1089/cap.2014.2425 


\title{
Re: J Child Adolesc Psychopharm 23:208-213, 2013
}

\author{
Hans-Christoph Steinhausen and Dorte Helenius, "The Association Between Medication \\ for Attention-Deficit/Hyperactivity Disorder and Cancer," J Child Adolesc Psychopharm 23:208-213, 2013.
}

\section{The authors have submitted the following correction to their article:}

N OUR RECENT PUBLICATION, we analyzed the risk of having a diagnosis of cancer in attention-deficit/hyperactivity disorder (ADHD) patients receiving various medications or no medication. Unfortunately, our previous risk analysis (Table 3 in the article) was incorrect and did not use the data from the prescription register in the most suitable way. The prescription database was initiated on January 1, 1995. However, in our previous analyses patients became at risk at birth and switched medical status whenever they received medication after January 1, 1995. This means that a number of cases with an unknown cancer status before January 1, 1995 may have been included in the no medication subgroup during their whole observation period. Furthermore, we assumed that individuals were without medication until January 1, 1995.

In the revised analysis we have used additional data from the Danish National Patient Registry on the occurrence of cancer in the period 1977-1993. When reanalyzing the data by Cox regression we followed the individuals from January 1, 1995 or date of birth if born later than this date and included treatment as a time-varying covariate. This follow-up ended December 31, 2009. Therefore, all cases having a diagnosis of cancer or dying before January 1, 1995 were not included in the survival analysis. Furthermore, the model was stratified on year of birth (divided into before, in, or after 1994) and sex because otherwise the proportional hazard assumption was not fulfilled.

A total of $N=60$ cases were diagnosed with cancer after January 1, 1995. The revised risk model revealed the following findings. Receiving methylphenidate (MPH) was associated with $89 \%$ decrease in the risk of cancer compared to the no medication subgroup (HR: 0.11, 95\% CI: 0.01-0.82) and mixed medication (MX) (including MPH as the largest component) was associated with $83 \%$ decrease in the risk of cancer compared to the no medication subgroup (HR: 0.17, 95\% CI: 0.06-0.50). Finally, receiving antidepressants (AD) was associated with an almost threefold increased risk of cancer in comparison to the no medication subgroup (HR: 2.70, 95\% CI: 1.04-7.01). Although receiving antipsychotics (AP) was associated with a twofold risk of cancer, this result was not significant (HR: 1.99, 95\% CI: 0.46-8.59). When excluding the MX subgroup from the analyses leaving $N=54$ cases occurring from January 1, 1995 onwards, the protective MPH subgroup effect remained significant (HR: $0.09,95 \%$ CI: 0.01-0.64) whereas the $\mathrm{AD}$ and $\mathrm{AP}$ subgroup effects were not significant anymore $(A D$ : HR: 1.88 , 95\% CI: 0.73-4.85; AP: HR: $1.52,95 \%$ CI: 0.35-6.53). The revised survival analysis based on the newly defined observation period is shown in Figure 1.
The comparability of the groups under treatment is fundamental to the investigation of treatment effects. Confounding by indication has been described as the most important limitation of observational studies. Among others, prognostic factors may influence treatment decisions and bias the estimates of the treatment effects. Controlling for known prognostic factors may reduce this bias but it is always possible that an overlooked or unknown factor may have exerted an effect. To avoid this bias, we calculated propensity scores based on age at diagnosis of ADHD (divided into five groups with equal frequencies), sex, year of birth (divided into five groups with equal frequencies), and comorbid disorders in additional analyses controlling for underlying systematic patterns. Comorbid disorders diagnosed according to ICD-10 from 1994 onwards were considered in major groups (main and secondary F-codes of the ICD-10; the F9 main group did not include F90 since all patients had this disorder) only prior to medication in the medicated groups and over the total observation period until cancer or date of censuring in the non-medicated subgroup. The results were in accordance with the analyses reported above. However, the additional variables in the propensity scores analysis may still have been too limited to detect any underlying systematic similarities in the treated groups.

In conclusion, both MPH and MX were protective of cancer while $\mathrm{AD}$ was associated with an almost threefold risk of cancer in comparison to the no medication subgroup. Excluding the MX

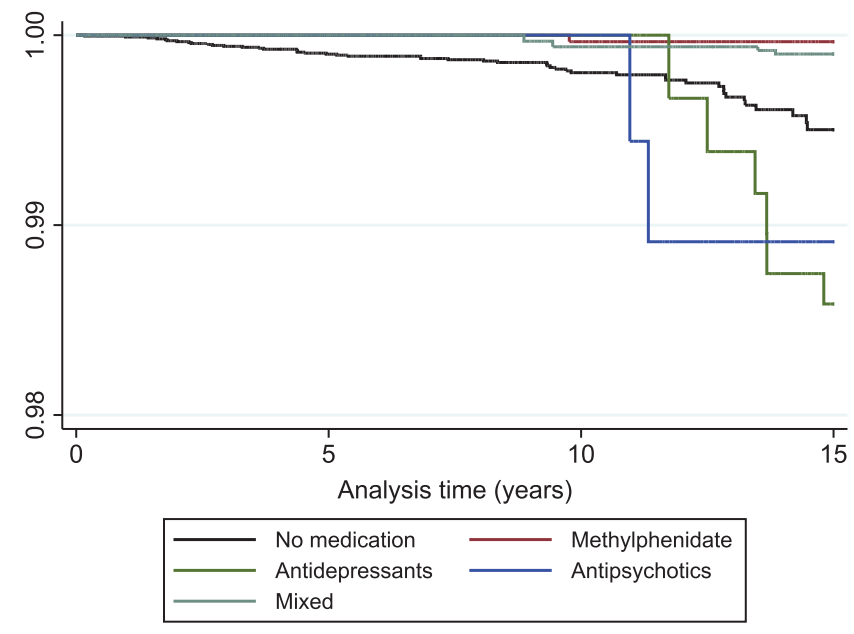

FIG. 1. Revised survival analysis findings (Kaplan-Meier curves) for various drug subgroups. A color version of the figure is available in the online correction at www.liebertpub.com/jcap. 
subgroup showed the same significant protective relation between MPH and cancer while the effects of AD and AP decreased and were not significant anymore. These findings imply that MPH was not only no significant risk factor of cancer as found in the previous analysis but rather a protective factor. The protective effect of MX was most probably also driven by the large MPH component in it. Furthermore, the impact of AD as a risk factor was limited by the large confidence intervals in the analysis including MX and by its lacking significance when excluding MX. 